\title{
A HOMOLOGICAL CHARACTERIZATION OF STEINITZ RINGS
}

\author{
HELMUT LENZING
}

Abstract. A ring $R$ (associative with an identity) is called a right Steinitz ring if any linearly independent subset of a free right $R$-module $F$ can be extended to a basis of $F$. Steinitz rings have been investigated in a recent paper of Chwe and Neggers. In this note it is shown that the right Steinitz rings are exactly the right perfect, local rings.

1. Preliminaries. $R$ denotes an associative ring with an identity, and all modules are unitary right $R$-modules. Obviously $R$ is a right Steinitz ring iff, for any free right $R$-module $F$, and any free submodule $U$ of $F, F / U$ is again free (and $U$ a direct summand of $F$ ). Every projective $R$-module $P$ over a right Steinitz ring $R$ is free: Let $P$ be a direct summand of a free module $F$ of infinite rank, then $F \cong P$ $\oplus F$ by a standard argument. Therefore $P \cong P \oplus F / F$ is free. Consequently $R$ is a right Steinitz ring iff any right $R$-module of finite homological dimension is free.

The Jacobson radical of $R$ is denoted $J=J(R) . R$ is local if $R / J$ is a division ring. The right finitistic projective dimension $\operatorname{rFP}(R)$ is defined in the sense of H. Bass [1, p. 478].

2. Results. The following theorem characterizes right Steinitz rings.

THEOREM. For any ring $R$ the following statements are equivalent:

(a) Any linearly independent subset of a free right $R$-module $F$ can be extended to a basis of $F$ by adjoining elements of a given basis.

(b) $R$ is a right Steinitz ring.

(c) Any countable linearly independent subset of a (countably generated) free right $R$-module $F$ can be extended to a basis of $F$.

(d) $R$ is right perfect and any finitely generated projective right $R$ module is free.

(e) $R$ is a right perfect, local ring.

Proof. We will give a cyclic proof. (a) $\Rightarrow(\mathrm{b})$ and $(\mathrm{b}) \Rightarrow(\mathrm{c})$ : Obvious. $(c) \Rightarrow(d)$ : Following Bass [1], for any sequence $\left\{a_{n}\right\}_{n=0}^{\infty}$ of elements of

Received by the editors June 22, 1970.

AMS 1969 subject classifications. Primary 1640, 1690; Secondary 1505.

Key words and phrases. Perfect ring, local ring, Steinitz ring, linearly independent subset, basis, finitistic projective dimension. 
$R$ we consider a free right $R$-module $F$ with basis $\left\{x_{n}\right\}_{n=0}^{\infty}$ and the submodule $G$ of $F$ generated by $\left\{x_{n}-x_{n+1} a_{n}\right\}_{n=0}^{\infty}$. Since $G$ is free, $G$ is a direct summand of $F$. Therefore the chain $\left\{R a_{n} \cdots a_{0}\right\}_{n=0}^{\infty}$ of principal left ideals terminates [1, Lemma 1.3]. Thus, $R$ is right perfect. By the argument preceding the theorem any countably generated projective right $R$-module is free.

(d) $\Rightarrow(\mathrm{e})$ : We shall now prove that $R / J$ is a division ring. Suppose $N$ is any right ideal of $R / J$. Since $R / J$ is semisimple $\operatorname{artinian}, N$ is a direct summand of $R / J . J$ is a nil ideal; therefore idempotents may be lifted modulo $J$, and $N$ has the form $D / D J$ with $D$ a direct summand of $R$. Since $D$ is free, $N$ is a free right ideal of the artinian ring $R / J$. Thus, $N=0$ or $N=R / J$.

(e) $\Rightarrow$ (a): Since $R$ is right perfect, the right annihilator of $J$ is nonzero [2, Corollary 3]. Since $R$ is local, any proper left ideal of $R$ is contained in $J$, consequently has nonzero right annihilator. Thus $\operatorname{rFPD}(R)=0[1$, Theorem 6.3].

For any $R$-module $E$ the $R / J$-module $E / E J$ is denoted by $\bar{E}$; for $x \in E$ the corresponding element of $\bar{E}$ is $\bar{x}$. Suppose now that $B$ is a basis of the $R$-module $F$ and $S$ a linearly independent subset of $F$. Since $\operatorname{rFPD}(R)=0$, the submodule $G$ generated by $S$ is a direct summand of $F$. Therefore $\bar{S}=\{\bar{s}: s \in S\}$ is a linearly independent subset of $\bar{F}$. Since $R / J$ is a division ring and $\bar{B}$ a basis of $\bar{F}$, there is a subset $T$ of $B(T \cap S=\varnothing)$ such that $\bar{S} \cup \bar{T}$ is a basis of $\bar{F}$. The proof of (e) $\Rightarrow$ (a) is completed by the following lemma.

Lемма. Suppose $R$ is right perfect, $F$ is a projective right $R$-module and $B$ is a subset of $F . B$ is a basis of $F$ iff $\bar{B}$ is a basis of the $R / J$ module $\bar{F}=F / F J$.

Proof. Suppose $\bar{B}$ is a basis of $\bar{F}$. Let $G$ be the submodule of $F$ generated by $B$. Clearly $\bar{G}=\bar{F}$; i.e. $(F / G) J=F / G$. Therefore $F=G$ since $J$ is right $T$-nilpotent $[1$, p. 473$]$. Now, if $H$ is a free right $R$-module with basis $B$, the identity map on $B$ induces an epimorphism $u: H \rightarrow F$ with kernel $N$. Since $0 \rightarrow N \rightarrow H \rightarrow F \rightarrow 0$ splits, the corresponding sequence $0 \rightarrow \bar{N} \rightarrow \bar{H} \rightarrow \bar{F} \rightarrow 0$ is exact. $\bar{u}: \bar{H} \rightarrow \bar{F}$ is an isomorphism by hypothesis, therefore $\bar{N}=0$. Again, since $J$ is right $T$-nilpotent, $N=0$. Thus, $u$ is an isomorphism and $B$ is a basis of $F$.

3. Remarks. There is another characterization of right Steinitz rings. Govorov [3] proved that $R$ is a right perfect, local ring iff every flat right $R$-module is free.

One may consider rings $R$ with the Steinitz property for finite sets, i.e. every finite linearly independent subset of a (finitely generated) 
free right $R$-module $F$ may be extended to a basis of $F$. A ring with this property need not be a right Steinitz ring. In fact, any semisimple artinian ring has the Steinitz property for finite sets.

On the other hand, a right perfect ring $R$ need not have the Steinitz property for finite sets. Otherwise, it would follow from [1, Theorem 5.4 and Theorem 6.3] that $\operatorname{rFPD}(R)=0$. The ring $R$ of all (upper) triangular $2 \times 2$-matrices over a field is right perfect and has $\operatorname{rFPD}(R)=1$.

H. Bass constructed a local ring $R$ which is right perfect, but not left perfect $[1$, p. 476]. Therefore, a right Steinitz ring need not be a left Steinitz ring.

\section{REFERENCES}

1. H. Bass, Finitistic dimension and a homological generalization of semi-primary rings, Trans. Amer. Math. Soc. 95 (1960), 466-488. MR 28 \#1212.

2. B.-S. Chwe and J. Neggers, On the extension of linearly independent subsets of free modules to bases, Proc. Amer. Math. Soc. 24 (1970), 466-470. MR 40 \#5652.

3. V. E. Govorov, Rings over which flat modules are free, Dokl. Akad. Nauk SSSR 144 (1962), 965-967 = Soviet Math. Dokl. 3 (1962), 836-838. MR 25 \#3076.

4. I. Kaplansky, Projective modules, Ann. of Math. (2) 68 (1958), 372-377. MR 20 \#6453.

Universität Bielefeld, Bielefeld, Germany 